\title{
Genetic influences on premature parturition in an Australian twin sample
}

\author{
Susan A Treloar ${ }^{1}$, George A Macones ${ }^{2}$, Laura E Mitchell ${ }^{3}$ and Nicholas G Martin ${ }^{1}$ \\ ${ }^{1}$ Queensland Institute of Medical Research and Joint Genetics Program, The University of Queensland, Brisbane, \\ Australia \\ ${ }^{2}$ The University of Pennsylvania Health System \\ 3The Children's Hospital of Philadel phia, Philadelphia, PA, USA
}

\begin{abstract}
We investigated possible genetic influences on women's liability to preterm birth, using data from a large sample of A ustralian female twin pairs. In a 1988-90 questionnaire survey, both members of 905 parous twin pairs (579 monozygotic and 326 dizygotic) reported on whether deliveries had been more than two weeks preterm. Tetrachoric twin pair correlations for first birth were $r_{M Z}=0.20 \pm 0.11$ and $r_{D z}=-0.03 \pm 0.14$, and for any birth were $r_{M z}=0.30 \pm 0.08$ and $r_{D Z}=0.03 \pm 0.11$. Best-fitting models to data contained only additive genetic influences and individual environmental effects. Heritability was $17 \%$ for preterm delivery in first pregnancy, and $\mathbf{2 7 \%}$ for preterm delivery in any pregnancy. In the former case, however, we could not reject a model without genetic influences. Although our data did not allow for differentiation of the varying aetiologies of premature parturition, results from this exploratory analysis suggest that further investigation of genetic influences on specific reasons for preterm birth is warranted. Twin Research (2000) 3, 80-82.
\end{abstract}

Keywords: Preterm birth, pregnancy outcome, twins, genetics

The risk of preterm birth has been found to be higher for women who were themselves born prematurely, ${ }^{1}$ suggesting a repeating pattern across generations. The relevance of genetic influences on prematurity had been highlighted by an earlier study using Old Order Amish records in Lancaster County, Pennsylvania, which suggested that prematurity was mostly related to the maternal genotype. ${ }^{2}$

In order to explore the possibility of genetic influences on a woman's liability to preterm births we have examined self-reported, retrospective data on premature birth provided by a large number of Australian, female twin pairs. These twins are members of the Australian National Health and Medical Research Council Twin Registry, and participated in a health and lifestyle survey conducted between 1988 and $1990 .^{3-6}$ Responses to this survey were obtained from both members of 1624 female monozygotic (MZ) and dizygotic (DZ) twin pairs aged 25 and older, for a pairwise response rate of $82 \%$. The study questionnai re included questions on their menstrual cycles and reproductive events, including specific obstetric factors relating to each delivery.

Premature delivery (preterm birth) was defined in the questionnaire as delivery 'over 2 weeks early',

Correspondence: Susan A Treloar, PhD, Queensland Institute of Medical Research, PO Royal Brisbane Hospital, Queensl and 4029, Australia. Tel: $61733620229 ;$ Fax: 61733620101; E-mail: sueT@qimr.edu.au

Received 15 November 1999; accepted 17 November 1999 rather than the standard definition of curtailment of pregnancy before the 37th week of gestation. We did not ask for number of weeks gestation. We investigated parous twins' independent reports of premature delivery of first baby, and also reports of whether any birth was premature using the $>2$ week criterion. Premature births may have been spontaneous or induced, vaginal deliveries or performed by Caesarean section. Data were also avai lable on these items, but analyses are not presented here.

Twin pair matrices of tetrachoric correlations, and corresponding asymptotic covariance matrices, were computed separately for MZ and DZ twin pairs, using the Windows version of PRELIS 2.20. ${ }^{7}$ These correlations apply appropriately to dichotomous categorical data where the underlying distribution is assumed to be continuous and normally distributed. Genetic models were fitted by the method of asymptotic least squares to estimate the contributions of additive genetic, shared and non-shared environmental effects, using $\mathrm{Mx} .{ }^{8}$ We proceeded by systematical ly testing the significance of dropping parameters in turn. In addition to the likelihood ratio $\chi^{2}$ test (LR), the A kaike Information Criterion (AIC, measured as $\chi^{2}-2 d f$ ) was used as an additional indicator of fit. On the grounds of parsimony the model with the least number of parameters that offered a fit not significantly worse than the full model was chosen. Data analysis methods are described more fully elsewhere. ${ }^{9}$ 
Both members of 905 parous twin pairs $(579 \mathrm{MZ}$ pairs and 326DZ pairs) answered the question concerning prematurity of their first and any subsequent birth. Ages of parous twins ranged from 25 to 87 years, and mean age was $43.94 \pm 0.25$ years. Mean ages of MZ twins (44.09years) and DZ (43.71) twins did not differ significantly. The prevalence of premature first birth in the sample was $9.4 \%$ of individuals in $\mathrm{MZ}$ pairs and higher at $12.9 \%$ in the DZ twins, giving an overall prevalence of $10.7 \%$. Premature parturition relating to any birth using the $>2$ week criterion was reported by $17.3 \%$ of individuals in MZ pairs and $21.6 \%$ of twins in DZ pairs. The percentage of nulliparae was the same $(26 \%)$ for both $M Z$ and $D Z$ individuals.

Non-parametric one-way median score tests showed that parous MZ and DZ twins did not differ significantly on median total pregnancies. However, they did differ on median number of full-term (vs aborted) pregnancies $(P<0.05)$ and of offspring $(P<0.01)$, the latter median being higher for $D Z$ twins (3) than for MZ twins (2). This suggested a higher prevalence of multiple births in the DZ twins. The median number of preterm babies reported by DZ twins was significantly higher than for MZ twins $(P<0.05)$, consistent also with a higher rate of multiple births.

The ratio of $\mathrm{MZ}$ to $\mathrm{DZ}$ twin pair correlations ( $>2: 1$ ) shown in Tablel suggests that genetic influences may be operating on premature parturition. ${ }^{10}$ For both prematurity variables, using the AIC as the indicator, best-fitting most parsimonious models contained only additive genetic variation (A) and individual environmental effects (E). For having had a premature delivery for any birth, the best fitting model (AIC $=-0.924$ ) included a heritability of $27 \%$. A model including non-additive genetic influences resulted in a better fit than a model containing shared environment (C), but was not a significant improvement on the AE model. A CE model containing no additive genetic variation was rejected on significance grounds. For premature delivery, the $A E$ model was again the model of choice (AIC $=-1.30$ ) with a heritability of $17 \%$; however, in this case a model containing no genetic influences could not be rejected on significance grounds.

Evidence suggests that in Australia the impact of changes in obstetric care that might influence premature labour were minimal up to $1975 .{ }^{11}$ We would

Table 1 Tetrachoric twin pair correlations for prematurity of first delivery and of any delivery

\begin{tabular}{lllll}
\hline & \multicolumn{2}{c}{ MZ pairs $(n=579)$} & \multicolumn{2}{c}{ DZ pairs $(n=326)$} \\
$\begin{array}{l}\text { Premature by } \\
>2 \text { weeks }\end{array}$ & $\begin{array}{l}\text { Twin pair } \\
\text { correlation }\end{array}$ & SE & $\begin{array}{l}\text { Twin pair } \\
\text { correlation }\end{array}$ & SE \\
\hline First birth & 0.20 & 0.11 & -0.03 & 0.14 \\
Any birth & 0.30 & 0.08 & 0.03 & 0.11 \\
\hline
\end{tabular}

not expect the impact of obstetric practices to differ for individuals on the basis of their zygosity. Increased prevalence of multiple births to DZ twins may have influenced pairwise concordance for preterm birth, but any effect would have been too small to detect in this sample. Data were retrospective and collected in 1988-90, before the widespread availability of assisted reproductive technologies, which are increasing the prevalence of non-monozygotic multiple births, and hence may influence preval ence of premature delivery.

We cannot unequivocally separate out the different aetiologies for preterm birth in our data. The aetiologies of spontaneous premature membrane rupture or preterm labour may well differ, and those of indicated preterm births relating to foetal or maternal morbidity clearly may vary even further. Retrospectivity may have influenced accuracy of recall of preterm delivery. Data on length of gestation or date of last menstrual period (LMP) were not available.

Furthermore, genetic heterogeneity may be implicated in the predisposing conditions. ${ }^{1}$ For example, the prevalence of pre-eclampsia, which may be associated with indicated premature delivery, is higher for first than subsequent births. If the aetiology is foetal in origin rather than maternal, which may well be the case, ${ }^{12}$ the effect may be to reduce the (maternal) twin pair correlations. This might suggest that the heritability we have observed is occurring in the spontaneous rather than the indicated preterm deliveries, but to test this hypothesis would require a very large sample indeed. It would be fascinating to know whether, for example, there is a genetic contribution to factors such as cervical incompetence per se. Nevertheless, our data do suggest that genetic influences may be important in influencing preterm birth, and further investigation of this hypothesis may well be warranted.

\section{Acknowledgements}

We thank the National Health and Medical Research Council of Australia for support (No.941177, No. 971232) and the twins for their co-operation.

\section{References}

1 Porter T, Fraser A, Hunter C, Ward R, Varner M: The risk of preterm birth across generations. Obstet Gynecol 1997; 90: 63-67.

2 Khoury M, Cohen B: Genetic heterogeneity of prematurity and intrauterine growth retardation: clues from the Old Order Amish. Am J Obstet Gynecol 1987; 157: 400-410. 
(1) Genetic influences on preterm birth in Australian twin mothers

82

3 Do K-A, Trel oar SA, Pandeya N, Purdie D, Green A, Heath AC, Martin NG: Predictive factors of age at menopause in a large Australian twin study. Hum Biol 1998; 70: 1073-1091.

4 Heath $A$, Cloninger $C$, Martin N: Testing a model for the genetic structure of personality: A comparison of the personality systems of Cloninger and Eysenck. J Pers Soc Psychol 1994; 66: 762-775.

5 Trel oar S, Martin N, Heath A: Longitudinal genetic analysis of menstrual flow, pain and limitation in a sample of Australian twins. Behav Genet 1998; 28: 107-116.

6 Treloar S, Martin N, Dennerstein L, Raphael B, Heath A: Pathways to hysterectomy: insights from longitudinal twin research. Am J Obstet Gynecol 1992; 167: 82-88.

7 Jöreskog K, Sörbom D: PRELIS 2.20 for Windows. Scientific Software International Inc: Chicago, 1998.
8 Neale M: Mx: Statistical Modeling. Department of Psychiatry: MCV, Richmond, VA, 1997.

9 Neale M, Cardon L: Methodology for Genetic Studies of Twins and Families NATO ASI Series. Kluwer Academic Publishers: Dordrecht, 1992.

10 Heath AC, Martin NG, Eaves LJ, Loesch D: Evidence for polygenic epistatic interactions in man? Genet 1984; 106: 719-727.

11 Stanley F: Perinatal outcome in Western Australia, 1968 to 1976. Perinatal mortality and birthweight. Med J Aust 1981; 1: 370-374.

12 Thornton J, Macdonald A: Twin mothers, pregnancy hypertension and pre-eclampsia. Br J Obstet Gynaecol 1999; 106: 570-575. 